\title{
Tipología de estrategias campesinas en la caficultura orgánica de la Sierra Madre de Chiapas
}

\section{Typology of peasant strategies in organic coffee farming in the Sierra Madre de Chiapas}

\author{
Yair Merlín-Uribe ${ }^{1 *}$, Fabien Charbonnier ${ }^{1}$, Armando Contreras-Hernández ${ }^{2}$, Obeimar Balente Herrera \\ Hernández ${ }^{1}$, Lorena Soto-Pinto ${ }^{1}$ \\ ${ }^{1}$ El Colegio de la Frontera Sur. Carretera Panamericana y Periférico Sur S/N, CP. 29290. San Cristóbal de las Casas, Chiapas, \\ México. \\ ${ }^{2}$ Instituto de Ecología A.C., Carretera Antigua a Coatepec, 351, Congregación El Haya, CP. 91070. Xalapa, Veracruz, México. \\ *Autor de correspondencia: yairmerlin7@gmail.com
}

Artículo científico recibido: 04 de enero de 2018 aceptado: 04 de mayo de 2018

RESUMEN. En la caficultura campesina existe un gradiente de estrategias productivas determinadas por factores funcionales. El objetivo del estudio fue construir una tipología de estrategias de manejo del cafetal de 87 caficultores de la Sierra Madre de Chiapas. Se utilizó una base de datos generada por una organización de caficultores orgánicos. Por medio de una clasificación jerárquica ascendente se identificaron tres tipos de estrategias, definidas por el sexo, edad y nivel de intensidad en el uso de factores productivos. El grupo más intensivo lo constituyeron mujeres que hacen uso de mano de obra permanente de origen externo. Ver el papel de la mujer y de los jóvenes en las estrategias productivas y de supervivencia en la caficultura campesina puede hacer efectiva la orientación y uso de recursos por las organizaciones de caficultores y mejorar el diseño de políticas públicas.

Palabras clave: Intensificación, café, mujeres, jóvenes, migración

ABSTRACT. In peasant coffee farming there is a gradient of productive strategies determined by functional factors. The objective of the study was to build a typology of coffee crop management strategies used by 87 coffee farmers in the Sierra Madre de Chiapas. A database generated by an organic coffee farmers' organization was used. By means of an ascending hierarchical classification, three types of strategies were identified, defined by sex, age and level of intensity in the use of productive factors. The most intensive group consisted of women who make permanent use of the labor of external origin. Seeing the role of women and youth in productive and survival strategies in peasant coffee farming can make the orientation and use of resources by coffee farmers' organizations effective and improve the design of public policies.

Key words: Intensification, coffee, women, youth, migration

\section{INTRODUCCIÓN}

Los estilos de agricultura se definen por un complejo repertorio de estrategias, acordes con los recursos que tienen los agricultores a su alcance (Nielsen et al. 2013). Se distinguen los agricultores agroempresariales y los campesino, entre ellos hay un gradiente que constituye la diversidad de formas de agricultura (Parmentier 2014). Para el agroempresario, los principales objetivos son la rentabilidad y los mercados (Nigh 2010). Mientras que el campesino prioriza la provisión de alimentos y materiales para el hogar (Edelman 2013). Ambos tipos de agricultores deciden la cantidad y periodicidad de los factores de producción, mismos que determinan su intensidad productiva (Nigh 2010). En este sentido, la toma de decisiones difiere con la lógica de producción y constituye un factor funcional que determina las estrategias de manejo y la estructura del sistema (Edwards-Jones 2007). La intensidad agrícola se refiere al radio entre los factores de producción y los productos por unidad de 
área o tiempo (Ruiz-Martinez et al. 2015). Dichos factores no son vinculantes a un estilo de agricultura, debido a que son relativos al sistema (Erb et al. 2013). En el contexto campesino, el trabajo familiar, la tierra y el capital monetario y social, son los factores de producción primarios, mientras que la diversificación económica, las remesas y los programas de gobierno se relacionan con estrategias de supervivencia (Nigh 2010, Ayala-Carrillo et al. 2014). A su vez las estrategias de supervivencia se relacionan con los niveles de intensidad productiva (Barham y Weber 2012).

La caficultura es una actividad económica de relevancia mundial, millones de personas obtienen sus ingresos del café (Lewin et al. 2004). Se estima que el $90 \%$ del café en el mundo se cultiva en pequeñas superficies, lo que genera un gradiente multidimensional heterogéneo (ICO 2017). En México, la parcial cobertura técnica del extinto Instituto Mexicano del Café junto con la liberalización comercial y la proliferación de certificaciones de producción, aumentaron la heterogeneidad socioambiental y económica en las regiones productoras de café (Hernández-Martínez et al. 2009). Entre el 2011 y 2015 brotes sucesivos de la roya del café (Cerda et al. 2017b) y la adopción de variedades resistentes a esta enfermedad reconfiguraron la caficultura campesina del sur de México (COMCAFE 2013).

En Chiapas el cultivo de café es determinante para el sustento de la población, pero se caracteriza por ser de bajo uso de insumos agroindustriales, lo que facilitó la adopción de la caficultura orgánica (Martínez-Torres 2004). El estado es el escenario de Organizaciones Cafetaleras campesinas (OC), por medio de éstas se acorta la cadena de valor y permite a los caficultores obtener mayores ingresos (Naylor 2017). Una de las orgnizaciones regionales representativa fue la Federación Indígena Ecológica de Chiapas (FIECH), compuesta por 18 organizaciones locales de primer nivel y 3023 caficultores certificados o en proceso de certificación. La FIECH operó en 21 municipios del estado, durante su funcionamiento, contó con los certificados NOP (CERTIMEX) y FLO-Cert (Comercio justo).
Se han propuesto diversas tipologías para abordar la complejidad relacionada con los estilos de manejo en la caficultura mexicana, se distinguen en tres enfoques: las relacionadas con la estructura de la vegetación (Moguel y Toledo 1999, Soto-Pinto et al. 2015), las relacionadas con los recursos y tecnología agrícola (Guadarrama-Zugasti, 2008) y las relacionadas con la biodiversidad (Mas y Dietsch 2003, Hernández-Martínez et al. 2009). Estos estudios proporcionan información relevante, pero al enfocarse en la racionalidad de la caficultura convencional-empresarial y la dimensión ambiental, omiten temas sociales de la caficultura orgánica campesina. Por lo anterior, el objetivo del estudio fue construir una tipología del manejo del cafetal y un índice de intensidad en la producción de café en el contexto campesino.

\section{MATERIALES Y MÉTODOS}

El área de estudio se ubica entre la Sierra Madre de Chiapas y la llanura costera (92 ${ }^{\circ} 47^{\prime}$ $06 "$ LN, $15^{\circ} 28^{\prime} 15^{\prime \prime}$ LE) (Figura 1). Los climas predominantes son cálido húmedo $(\mathrm{Am})$ y semicálido húmedo $(\mathrm{ACm})$ con lluvias en verano. La precipitación anual oscila entre los 2000 y 4000 mm (INEGI 1984), con temperatura media mínima y máxima anual de 18 a $35^{\circ} \mathrm{C}$ (INEGI 2008). Los suelos predominantes son Luvisol, Regosol y Phaeozen (CONAFOR 2007). El principal uso de suelo es agricultura de temporal con remanentes de selva alta perennifolia y de bosques de pino (INEGI 2015). El área se localiza en las delegaciones cafeteras de Tapachula y Motozintla, ambas importantes para la caficultura con las condiciones climáticas óptimas para el cultivo de las variedades Typica, Bourbon, Maragogipe y Mundo Novo, apreciadas por su calidad en taza (Pérez-Portilla et al. 2011). En estas delegaciones se concentra el $23 \%$ de los caficultores y el $37 \%$ de la superficie de café del estado, cerca del $50 \%$ de los caficultores producen en parcelas de menos de 1 ha (COMCAFE 2013).

\section{Obtención de la información}

Los datos se obtuvieron entre octubre de 2015 


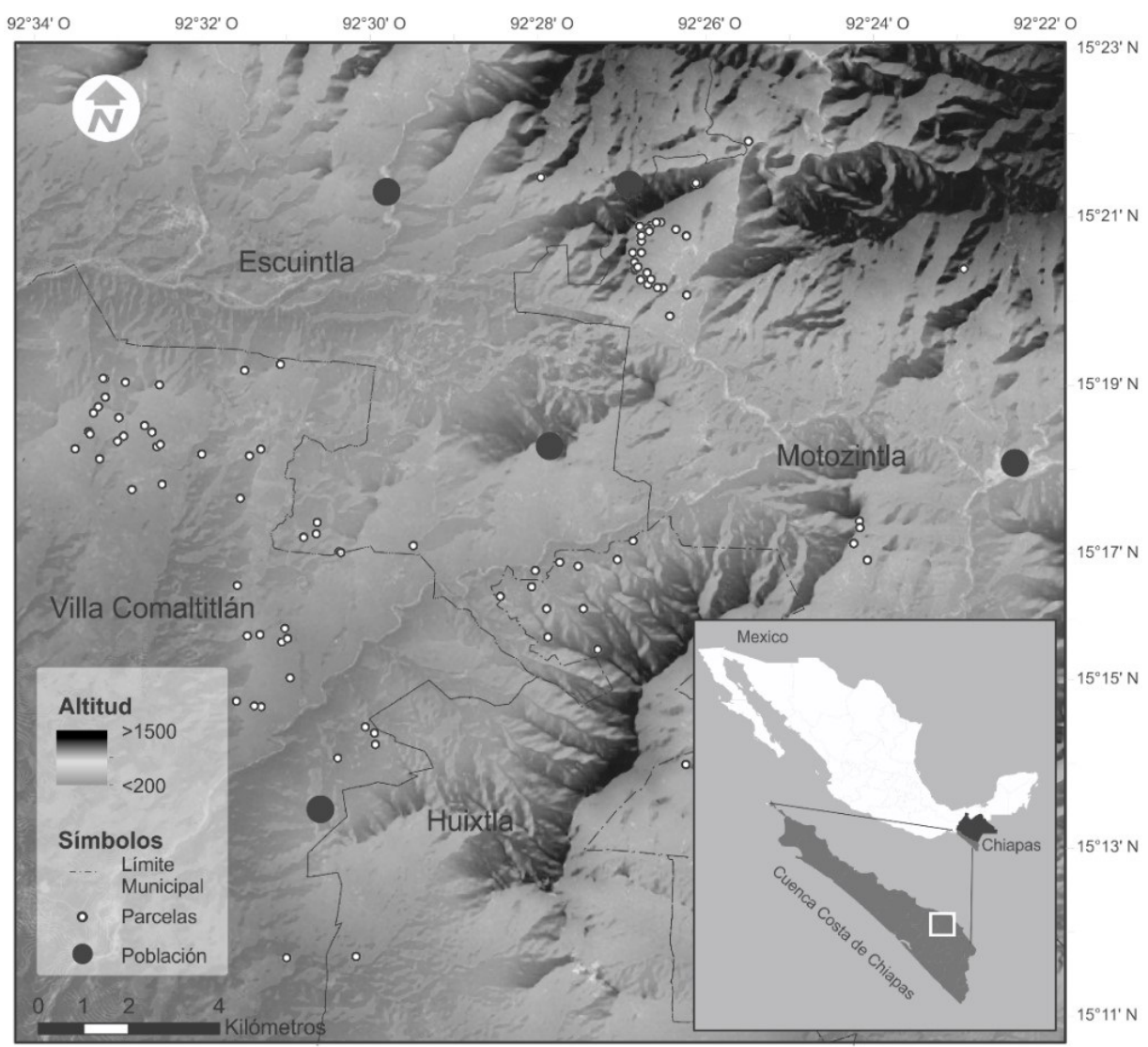

Figura 1. Área de estudio, la escala de gris del relieve indica altitud.

y febrero de 2016 por los técnicos de la FIECH, por medio de un muestreo dirigido a 93 caficultores. La información se obtuvo como parte de los datos obtenidos para la certificación orgánica que anualmente exige la FIECH a sus agremiados. Se empleó un formulario prediseñado por la FIECH para entrevistar a los productores en la parcela. La información se registró en la aplicación ODK Collect para dispositivos móviles con sistema Android (Brunette et al. 2017), para reemplazar los formularios en papel y facilitar la captura de información. La información se sometió a controles de calidad por el personal de la organización y la Certificadora CERTIMEX. A partir de la validación, la FIECH generó la base de datos (BDD) con información de identificación del caficultor, características de la parcela y manejo agronómico del cafetal. La información se analizó con autorización de la FIECH como parte del convenio de colaboración con el Colegio de la Frontera Sur. De forma complementaria, entre el 2015 y 2017, se realizó observación participante (Kawulich 2005), durante las visitas a las parcelas de los caficultores agremiados y en los talleres con técnicos y directivos de la Organización.

\section{Selección de variables y clasificación}

La base de datos se sometió a depuración supervisada para satisfacer los criterios de validez, precisión, integridad, consistencia y uniformidad (De Vaus 2014). Se seleccionaron las variables mediante los siguientes criterios: (a) que constituyeran factores de producción en el cultivo de café; (b) se seleccionaron las variables con coeficiente de variación superior al 50\% (OECD 2008),y (c) y baja multicolinealidad (Spearman $\alpha=0.05$ ). Como resultado se obtuvieron 87 casos con 25 variables 
(19 categóricas y 6 numéricas). Las variables categóricas se ponderaron de acuerdo a su peso en el aporte a la variable: 0 significó bajo peso y 1 alto peso. Las variables con más de dos categorías se ponderaron a partir del número de categorías dividido entre uno con asignación de pesos (Tabla 1 ). Se estandarizaron los valores con la desviación estándar sin sesgo (n-1), para evitar el efecto de sesgo en tamaños de muestra pequeños (Addinsoft 2016). Posteriormente, se realizó una Clasificación Jerárquica Ascendente (CJA), con la disimilitud de Spearman como distancia entre observaciones y el promedio no ponderado par-grupo como método de aglomeración (Sokal y Michener 1958). El truncamiento para el número de conglomerados se determinó de forma automática por medio de la entropía (Everitt et al. 2011). La precisión de la clasificación se comprobó con un Árbol de Clasificación y Regresión (ACR), con ajuste de Bonferroni $(p<0.05)$ (Kass 1980). Se validó estadísticamente la existencia de los grupos con una prueba de KW (KruskalWallis, KW $\alpha<0.05)$ con comparación múltiple por pares de Dunn y corrección de Bonferroni ( $p$ $<0.05)$. Con fines confirmatorios, la columna de clases generada por la CJA se usó como indicador de agrupación en un Análisis de Componentes Principales (ACP) tipo Spearman.

\section{Índice agregado de intensidad}

Se construyó un índice agregado con las consideraciones metodológicas de la OECD (2008), Hernández-Martínez et al. (2009) y Cerda et al. (2017a). El criterio de selección de variables fue que correspondieran a factores de producción en el contexto campesino. Para construir el índice se utilizaron las variables: temporalidad de la mano de obra, tipo de mano de obra, poda de cafetos, control de plagas y enfermedades, tenencia de semillero y despulpadora, y manejo de la pulpa (Tabla 1). Se realizó un análisis factorial (AF) con el método de correlaciones policóricas y extracción de factores principales. El índice se compuso de las puntuaciones factoriales del Primer Factor (FA1) (Jolliffe 2002, OECD 2008). Para comprobar el poder explicativo del índice, se realizó una regresión lineal múltiple con simulaciones de Montecarlo, donde la variable dependiente fue el FA1 y las explicativas las variables de producción. Luego se determinó la significancia estadística de los valores del índice entre grupos, con una prueba de $\mathrm{KW}(\alpha<0.05)$ con comparación múltiple por pares de Dunn y corrección de Bonferroni $(p<0.05)$. Este índice se consideró como medida agregada del desempeño de las variables de trabajo por tipo de caficultor, si el índice tuvo valor 1 indicó que la labor se hizo por la mayoría del grupo. Los análisis se realizaron con el paquete R 3.3.3 y Excel Stat (Addinsoft 2016).

\section{Validación de campo}

La tipología y el índice de intensidad se validó en campo por consulta con cuatro técnicos expertos de la FIECH. Los criterios para considerarlos expertos fueron: ser originarios de la zona, tener amplia experiencia en la caficultura, conocer a la mayoría de los productores de la región y haber desempeñado el cargo por más de tres años en la organización. A los expertos se les presentó la tipología y solicitó describieran a detalle a los tipos resultantes. La información se capturó en un diario de campo para su posterior sistematización y análisis.

\section{RESULTADOS}

\section{Clasificación de las observaciones}

La clasificación de las observaciones sugirió tres grupos: el tipo 1 (T1) concentró el $28 \%$ de las observaciones, el tipo 2 (T2) el $12 \%$; y el tipo 3 (T3) el 58\%. El análisis ACR mostró que se alcanzó el $93.1 \%$ de precisión en la clasificación. El ACP confirmó la agrupación de las observaciones, la superposición de las elipses en el gráfico indica la existencia de similitud entre los grupos en algunas dimensiones, pero los centroides de los Componentes Principales (CP) 1 y 2 estuvieron lejanos, lo que indica que son grupos bien definidos (Figura 2).

Las variables que se correlacionan con el CP 1 fueron: poda de los cafetos (PodaCaf), edad del productor (EdaPrd) y número de parcelas (NoParc); con el CP 2 se relacionaron: temporalidad de uso de mano de obra (TempMO), tipo de mano de obra 


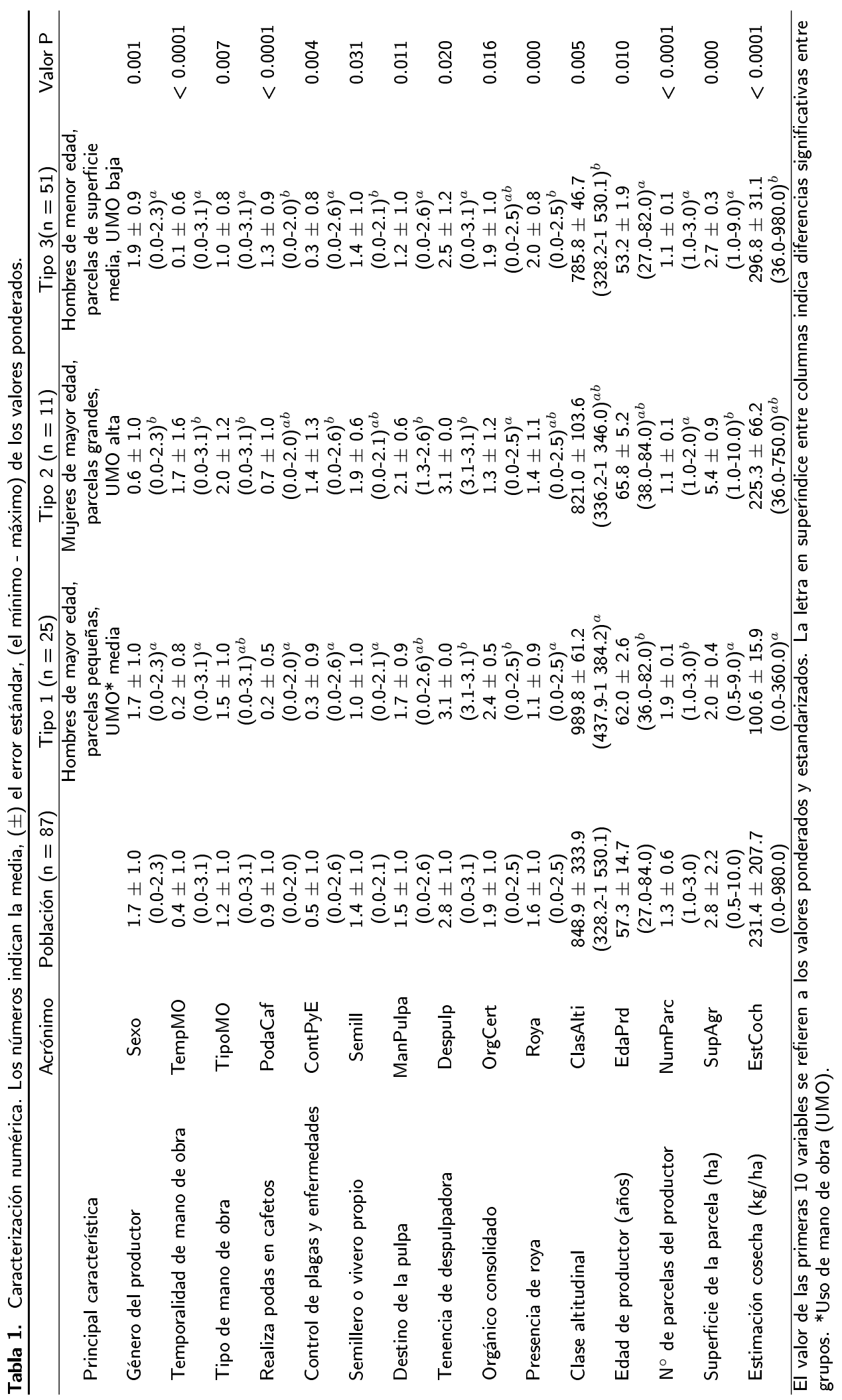




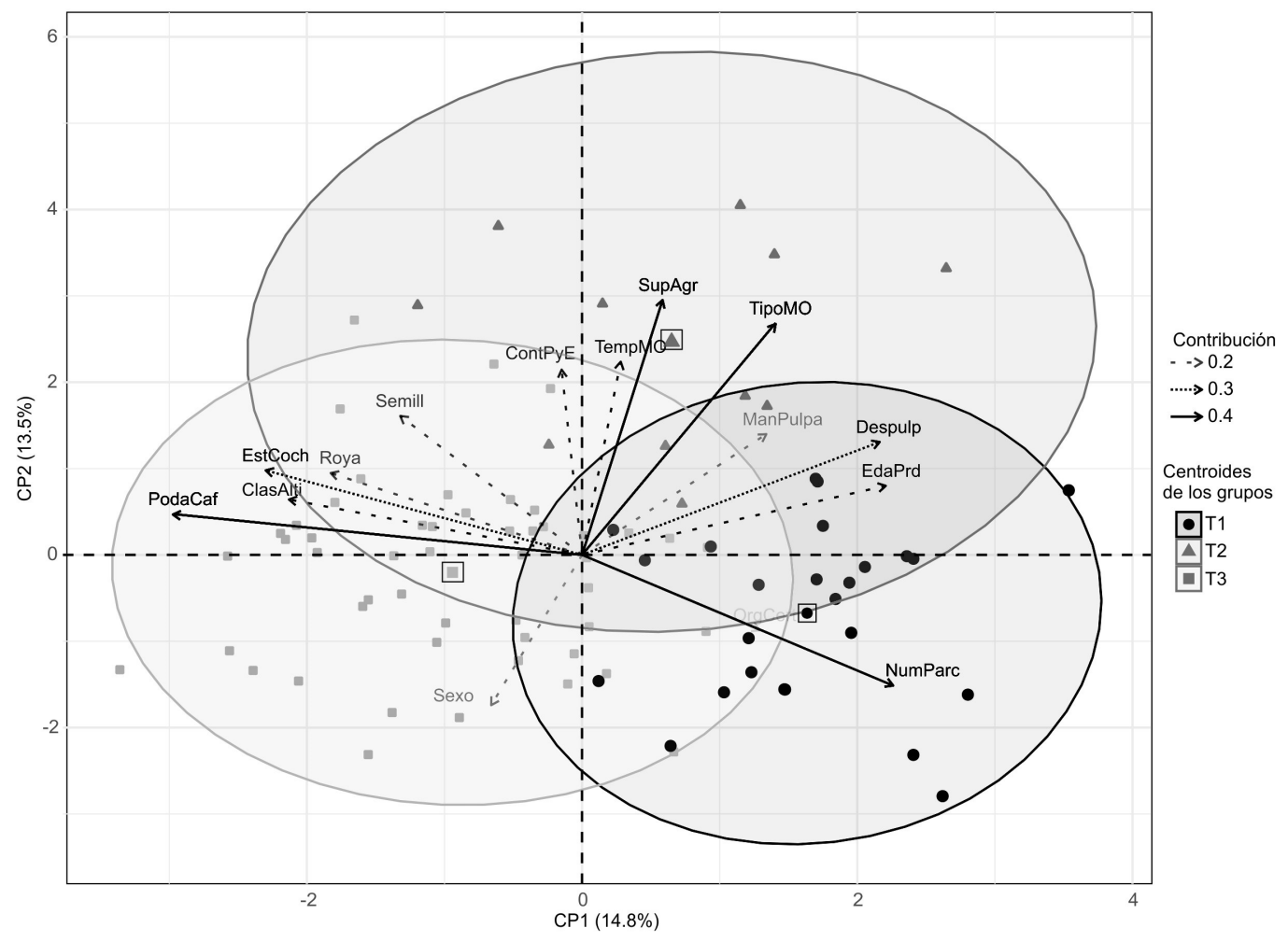

Figura 2. Biplot de correlación entre las variables y los componentes. El peso de la variable en la definición de los grupos se representa con el tono y la forma de los vectores. Cada observación se representa por un símbolo de acuerdo al tipo en el que se agrupa. Acrónimos: Sexo del productor (Sexo); Temporalidad de mano de obra (TempMO); tipo de mano de obra (TipoMO); Poda de cafetos (PodaCaf); Control de plagas y enfermedades (ContPyE); Tenencia de semillero (Semill); Manejo de la pulpa (ManPulpa); Tenencia de despulpadora (Despulp); Certificación orgánica (OrgCert); Incidencia de roya (Roya); Clase altitudinal (ClasAlti); Edad del productor (EdaPrd); Número de parcelas (NumParc); Superficie de la parcela (SupAgr) y Estimación de la cosecha (EstCoch).

(TipoMO) y superficie de la parcela (SupAgr). Con el CP 3 se correlacionó estatus de certificación (OrgCert); y con el CP 4 se correlacionó de forma negativa la variable sexo. Los tipos se asociaron a las variables que mayor varianza aportaron, el T1 se asoció con la EdaPrd, el NoParc, la Tenencia de despulpadora (Despulp) y el Manejo de la pulpa (ManPulpa); el T2 se asoció a la SupAgr, el TipoMO, la TempMO y la Tenencia de semillero (Semill). El T3 se asoció a la aplicación de PodaCaf, la Semill, la Estimación de la cosecha (EstCoch) y el sexo del productor (Figura 2).

\section{Tipos de estrategias}

Se encontraron diferencias estadísticas en 15 de las 25 variables evaluadas, el resultado confirmó la existencia de tres tipos de estrategias de manejo (Tabla 1). La descripción de los tipos se basó en las variables con mayor aporte a la varianza de los CP. Los caficultores del tipo T1 en su mayoría fueron hombres con edad promedio de 62 años, poseyeron más parcelas que el resto de los productores y tuvieron despulpadora propia. Casi la mitad de ellos usó la pulpa como abono e hicieron uso de mano de obra en nivel medio con respecto a los demás grupos, además de que la mayoría fueron caficultores con certificación orgánica. El $40 \%$ de las parcelas de este grupo se ubicaron en zonas con altitud superior a los 1530 msnm (Tabla 1, Figura 3). Las caficultoras del tipo T2 fueron en su mayoría mujeres con 


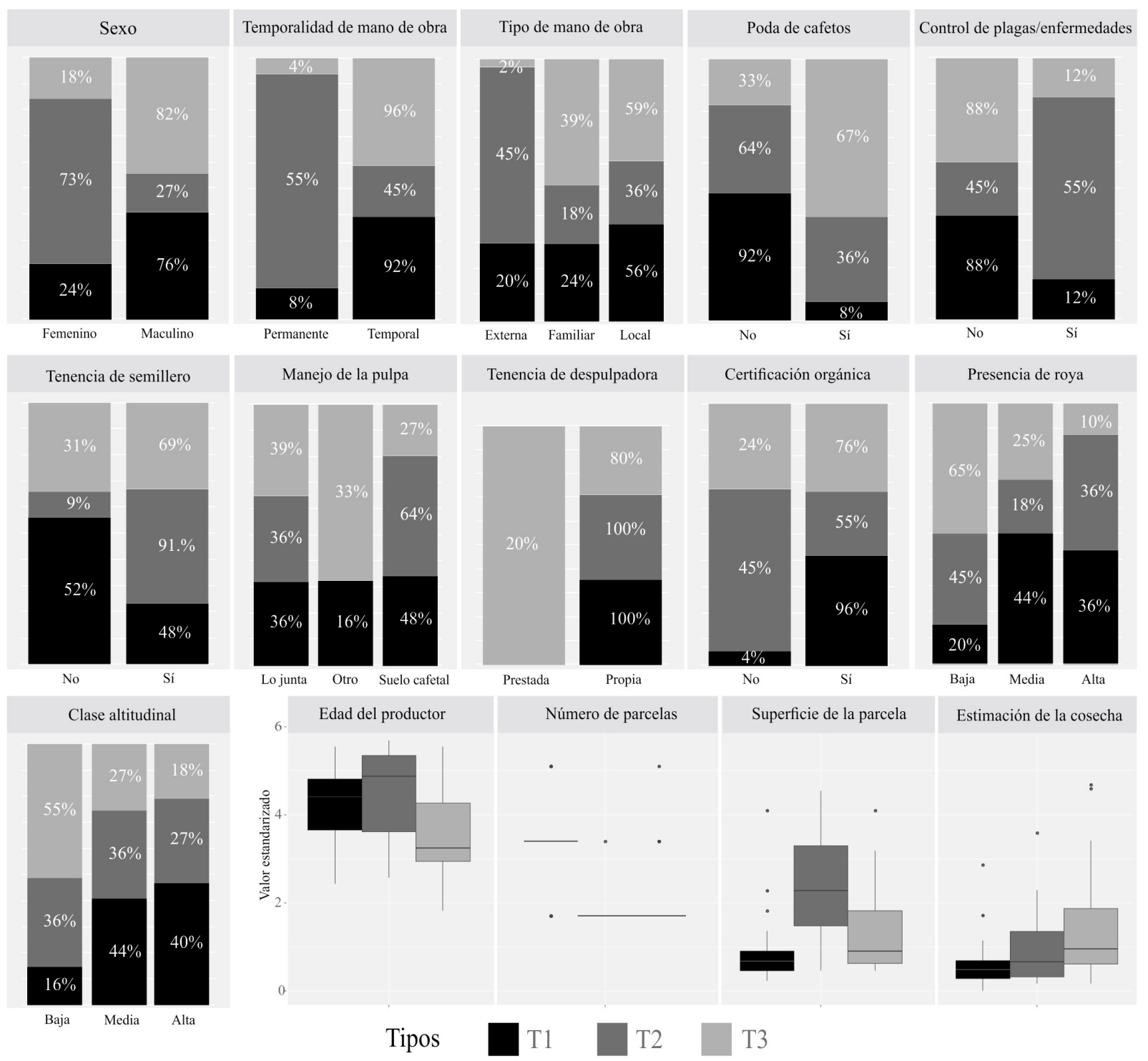

Figura 3. Caracterización de los tipos de manejo. Los valores de las barras son las frecuencias relativas de las variables categóricas. Los valores de los gráficos de caja se refieren al valor estandarizado de las variables numéricas.

edad promedio de 65 años, poseyeron las parcelas de mayor superficie, tuvieron semillero o vivero propio en mayor proporción e hicieron con mayor frecuencia control de plagas. También destacaron por hacer alto uso de mano de obra permanente de origen externo. Los caficultores del tipo T3, lo constituyeron en su mayoría hombres con edad promedio de 53 años, quienes tuvieron la menor edad; realizan poda en los cafetos, poco más de la mitad tuvieron semillero propio y la estimación de cosecha fue la mayor. Las parcelas de estos caficultores tuvieron los niveles más bajos de roya y utilizaron menor mano de obra que el resto. Destacó que el $55 \%$ de sus parcelas se ubicaron en zonas bajas $(<816$ msnm).

Índice agregado de trabajo

El análisis factorial reveló que el primer factor 
(FA1) explicó el $40 \%$ y el segundo (FA2) el $60 \%$ de la variación acumulada. La mayoría de las variables de trabajo: ContPyE, TipoMo, Semill, ManPulpa y Despulp estuvieron correlacionadas al FA1. Las variables TempMo y PodaCaf se correlacionaron de forma negativa al FA2. Las caficultoras del manejo T2 estan más relacionadas con las variables de trabajo que el resto de los tipos de caficultores, con excepción de la variable PodaCaf. Los parámetros de la regresión entre el FA1 y las variables explicativas indican que el índice agregado tuvo un alto poder explicativo. La prueba de KW indicó diferencias significativas entre grupos, lo que confirmó que estos tipos manejan el cafetal con diversos grados de intensidad. En este gradiente las caficultoras del T2 fueron las más intensivas en términos de trabajo en el cafetal (Figura 4).

\section{Descripción de los tipos por expertos}

Los expertos identificaron a productores con un manejo del cafetal coincidente con la tipología. Desde su perspectiva, el estado de salud y la edad del caficultor combinado con la falta de capital económico son en gran medida, factores limitantes en la producción. La edad y sexo del productor fueron útiles para los expertos, debido a que facilitaron la vinculación de los tipos a procesos de migración, posesión de conocimientos, capacidades físicas y económicas. En la descripción, coicidieron en los temas de recepción de remesas y apoyos gubernamentales como principales fuentes de capital para mantener el cafetal.

\section{DISCUSIÓN}

La validación de la clasificación indicó tres tipos de manejo del cafetal. El manejo T1 lo practicaron hombres de edad avanzada, en parcelas de pequeña superficie y uso de mano de obra medio; el manejo T2 lo hicieron mujeres de edad avanzada, con parcelas de mayor superficie y alto uso de mano de obra; y el manejo T3 lo practicaron los hombres de menor edad de la muestra en parcelas de superficie media con uso moderado de mano de obra. El índice agregado de trabajo indicó que las mujeres del
T2 fueron más intensivas en el manejo. Entre los hombres del T1 y T3 no se encontraron diferencias significativas.

Los caficultores del T1 fueron los que en mayor proporción tuvieron certificación orgánica. Este grupo fue el que menos labores de manejo realiza en el cafetal, lo que se refleja en baja estimación de cosecha y altos niveles de roya. Los expertos aclararon que este grupo son los experimentados, debido a que omiten labores porque la edad los limita, pero son los que hacen fuerte a la OC al mantener la certificación orgánica; este tipo de caficultor se enfoca más en la calidad que en la cantidad. Las caficultoras del T2 tienen edad avanzada, lo que pudo incidir en que fueran las que más dependan de mano de obra contratada. De acuerdo con los expertos, algunas de estas mujeres reciben remesas o apoyos de gobierno que invierten en el cafetal, también hay algunas mujeres que administran su propia parcela y venden su café, sin que el esposo o los hijos intervengan en la toma de decisiones. De acuerdo con los expertos los caficultores del T3 se enfocan en el volumen, lo que coincide con la contratación de mano de obra temporal, y realizan poda, actividad demandante de trabajo. De acuerdo con Hernández-Solabac et al. (2011) y Avelino et al. (2004) la realización de la poda incide de forma positiva en la productividad y la baja prevalencia de roya, lo que explica que los agricultores de manejo T3 tuvieron la mayor estimación de cosecha.

En general, el manejo del cafetal fue de baja intensidad productiva con limitado empleo de insumos o practicas orgánicas. Aun cuando se contrata mano de obra, las bajas frecuencias en las prácticas culturales indican que la mano de obra se limita al período de cosecha. La baja intensidad agroindustrial y alto uso de mano de obra temporal es característico de la caficultura campesina y responde a estrategias de supervivencia. Al respecto Martínez-Torres (2004) y López-López y CaamalCauich (2009), atribuyen la baja intensidad productiva a una relación negativa entre el costo y el beneficio en la producción de café, por lo que los caficultores distribuyen su fuerza de trabajo en otras actividades que complementan su ingreso. En tanto 


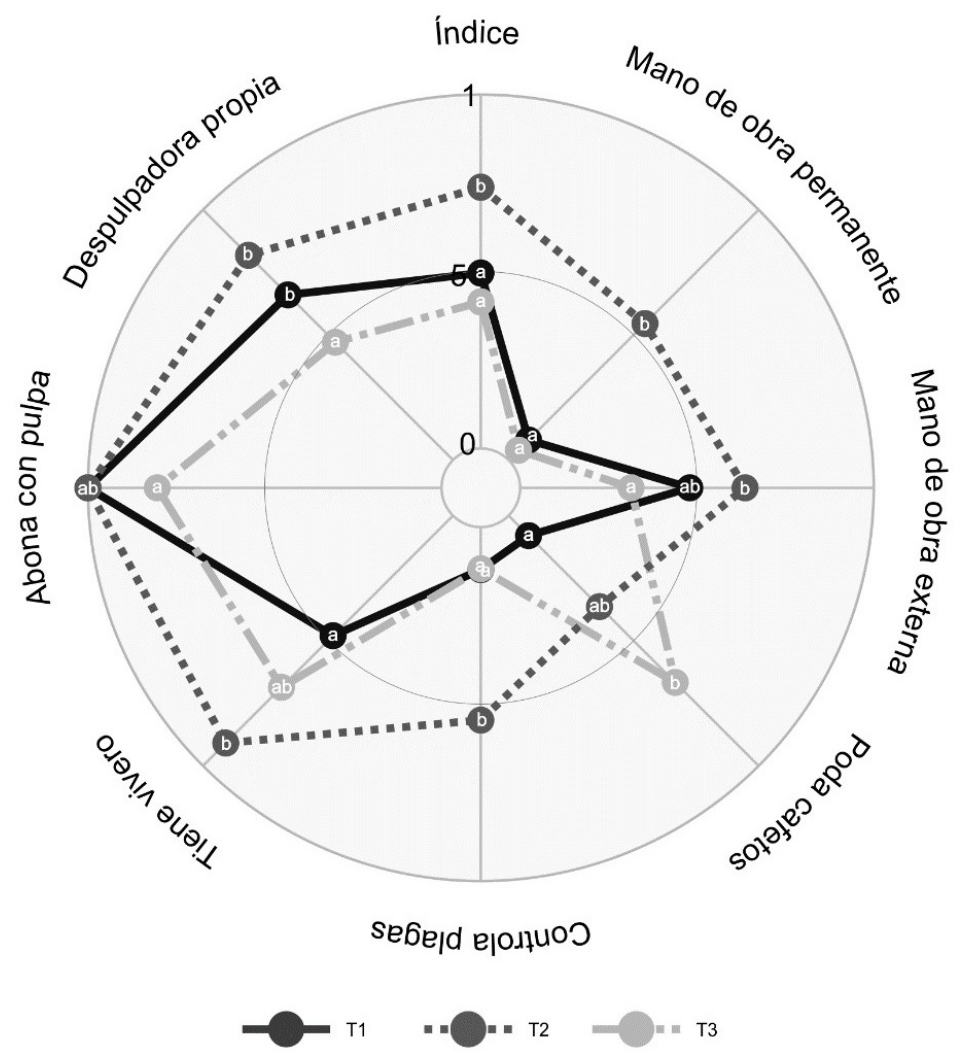

Figura 4. Desempeño de las variables de trabajo respecto a su tipo. EI contorno de la línea indica el tipo de estrategia. El índice es una medida agregada y ponderada de las siete variables. Las letras en los radios indican diferencias significativas.

que Aguilar-Støen et al. (2011) mencionan que los caficultores tienen experiencia en la fluctuación de los precios del café, asumen que por más que inviertan trabajo en el cafetal el ingreso es inestable, por lo que prefieren repartir su trabajo en otras actividades. Weber (2012) indica que tener una certificación orgánica y comercio justo posiciona a los caficultores en un estatus de ingreso superior al caficultor convencional, aún con un perfil de baja intensidad productiva. Otra estrategia común en los caficultores es el manejo múltiple de sus parcelas de café, lo que coincide con Sánchez-Juárez (2015), quien indica que los caficultores tienen parcelas con manejo convencional y otras con manejo orgánico.

Las variables sexo, edad y trabajo en el cafetal tienen influencia en la formación de los grupos, sin embargo, los expertos añadieron la migración, remesas y dinámica familiar como otros factores explicativos. En la literatura se vinculan las estrategias de supervivencia y producción campesina con la migración, las remesas y el comercio internacional de materias primas (Davis y Lopez-Carr 2014, RosalesMartínez et al. 2014). Para el café orgánico, estos factores influyen en la estructura de las familias y las comunidades. Al respecto, Méndez et al. (2010) y Rus y Rus (2014) concluyen que en hogares dedicados a la caficultura certificada, es común que los más jóvenes migren en busca de empleo, mientras que los padres se mantienen en la comunidad; lo que tiene un fuerte impacto en los roles de género, la dinámica familiar y la estructura de la población. Otro aspecto es la relación entre la certificación y la migración, al respecto Lewis (2005) y Jaffee (2014) reportan que los caficultores certificados bajo el es- 
quema de comercio migran más que los no certificados, los autores indican que el excedente económico de la certificación se usa para migrar. Al respecto Escamilla et al. (2005) encontraron que al menos un miembro de cada familia dedicada a la caficultura orgánica migra a los Estados Unidos, lo que coincide con el peso interpretativo que dieron los expertos a la migración en la tipología. La migración nacional e internacional de los jóvenes puede explicar la alta proporción de caficultores con más de 60 años dentro de la muestra.

Las mujeres adoptan una estrategia más intensiva, lo que se correlacionó con el origen y el período de contrato de la mano de obra. El caso de las mujeres no es aislado, varios estudios esclarecen el papel de la mujer en la caficultura, en contextos de recursos limitados, las mujeres son más intensivas en el trabajo que los hombres, incluso se encargan de las tareas que mayor valor agregan al café, lo que tiene un impacto positivo en el ingreso (Lyon et al. 2010, Cueva-Alegría 2016). Mientras que AguilarStøen et al. (2011) reportaron que los apoyos de gobierno y las remesas cumplen un papel indirecto en mantener los cafetales manejado por mujeres.

Las características de manejo del presente estudio, en comparación con los descritos por Guadarrama-Zugasti (2008) y Hernández-Martínez et al. (2009), demuestra que se trata de caficultores con labores, tecnología, insumos y rendimientos limitados. En el contexto campesino existe un gradiente de intensidad y estrategias productivas y de supervivencia que han sido ignoradas por las tipologías enfocadas a la caficultura empresarial. Al respecto Nigh (2010) y Vallema et al. (2015) argumentan que los campesinos con acceso limitado a insumos agroindustriales intensifican la producción por medio del trabajo y la organización. En el caso de los caficultores vinculados al sector orgánico, este proceso se ha dado por la conformación de cooperativas y la apropiación de la cadena de valor.
El enfoque utilizado en la clasificación comprobó la existencia de los tres tipos en 15 de las 25 variables empleadas lo que indica que puede existir un número indeterminado de estrategias de manejo en el resto de las variables. En futuras tipologías se recomienda realizar una segunda clasificación con las variables que no se ajusten a la estructura general.

\section{CONCLUSIONES}

Integrar variables estructurales y funcionales en las tipologías de estrategias de manejo del cafetal, provee información para comprender procesos socioambientales complejos. Considerar la racionalidad de la caficultura campesina y sus estrategias de producción mostró procesos subyacentes que intervienen en las estrategias de supervivencia. La tipología indicó el papel de las mujeres caficultoras y los procesos externos con los que se relacionan. La validación de la tipología por expertos proporcionó información para vincular las estrategias de manejo con las estrategias de supervivencia. Los resultados aportan información que puede ser considerada en el diseño de programas técnicos y sociales por las organizaciones de caficultores.

\section{AGRADECIMIENTOS}

Al personal de la FIECH por facilitar la base de datos, a los caficultores que nos recibieron en sus hogares y parcelas, a los expertos de la organización por la interpretación de la tipología, a Raquel Bayarri por su asistencia en la redacción y a los revisores anónimos del escrito. Al financiamiento proporcionado por el proyecto Multidisciplinario y transversal: Innovación socioambiental en zonas cafetaleras para la reducción de la vulnerabilidad (MT \# 1106311262) de El Colegio de la Frontera Sur.

\section{LITERATURA CITADA}

Addinsoft (2016) XLSTAT, Data analysis and statistics software for Microsoft Excel. Ver. 2016.02. Addinsoft. New York, USA. 1446p 
Aguilar-Støen M, Angelsen A, Støle K-A, Moe SR (2011) The emergence, persistence, and current challenges of coffee forest gardens: A case study from Candelaria Loxicha, Oaxaca, Mexico. Society and Natural Resources 24: 1235-1251.

Avelino J, Willocquet L, Savary S (2004) Effects of crop management patterns on coffee rust epidemics. Plant Pathology 53: 541-547.

Ayala-Carrillo M, Zapata-Martelo E, Suárez-San Román B, Nazar-Beautelspacher A (2014) Estrategias de reproducción familiar en las fincas cafetaleras del soconusco, Chiapas. Agricultura, Sociedad y Desarrollo 11: 401-423.

Barham B, Weber J (2012) The economic sustainability of certified coffee: Recent evidence from Mexico and Peru. World Development 40: 1269-1279.

Brunette W, Sudar S, Sundt M, Larson C, Beorse J, Anderson R (2017) Open Data Kit 2.0: A services-based application framework for disconnected data management. Proceedings of the 15th Annual International Conference on Mobile Systems, Applications, and Services. New York, USA. pp: 440-452.

Cerda R, Allinne C, Gary C, Tixier P, Harvey CA, Krolczyk L (2017a) Effects of shade, altitude and management on multiple ecosystem services in coffee agroecosystems. European Journal of Agronomy 82: 308-319.

Cerda R, Avelino J, Gary C, Tixier P, Lechevallier E, Allinne C (2017b) Primary and secondary yield losses caused by pests and diseases: Assessment and modeling in coffee. PLoS One 12: e0169133.

COMCAFE (2013) Programa institucional 2013-2018. Comisión para el Desarrollo y Fomento del Café de Chiapas. Tuxtla Gutiérrez, México. 34p

CONAFOR (2007) Estudio regional forestal de la UMAFOR 0707 Sierra, Chiapas. Comisión Nacional Forestal. México. 378p

Cueva-Alegría D (2016) The case of café femenino: The limitations of gender-conscious fair trade. World Development Perspectives 1: 1-3.

Davis J, Lopez-Carr D (2014) Migration, remittances and smallholder decision-making: Implications for land use and livelihood change in Central America. Land Use Policy 36: 319-329.

De Vaus D (2014) Surveys in social research. Routledge. New York, USA. 379p.

Edelman M (2013) What is a peasant? What are peasantries? A briefing paper on issues of definition. The Graduate Center. The City University of New York. Ginebra. Suiza. 18p

Edwards-Jones G (2007) Modelling farmer decision-making: concepts, progress and challenges. Animal Science 82: 783-790.

Erb KH, Haberl H, Jepsen MR, Kuemmerle T, Lindner M, Muller D, et al. (2013) A conceptual framework for analysing and measuring land-use intensity. Current Opinion in Environmental Sustainability 5: 464-470.

Escamilla P, Ruiz R, Díaz P, Landeros S, Platas R, Zamarripa C, et al. (2005) El agroecosistema café orgánico en México. Manejo Integrado de Plagas y Agroecología 76: 5-16.

Everitt B, Landau S, Leese M, Stahl D (2011) Cluster analysis. John Wiley \& Sons. London, UK. 330p.

Guadarrama-Zugasti C (2008) A grower typology approach to assessing the environmental impact of coffee farming in Veracruz, México. En: Christopher B, Méndez E, Gliessman S, Goodman D, Fox J (ed). 
Confronting the coffee crisis: Fair trade, sustainable livelihoods and ecosystems in Mexico and Central America. Massachusetts, USA. pp: 127-154.

Hernández-Martínez G, Manson RH, Hernández AC (2009) Quantitative classification of coffee agroecosystems spanning a range of production intensities in central Veracruz, Mexico. Agriculture, Ecosystems and Environment 134: 89-98.

Hernández-Solabac J, Nava-Tablada M, Díaz-Cárdenas S, Pérez-Portilla E, Escamilla-Prado E (2011) Migración internacional y manejo tecnológico del café en dos comunidades del centro de Veracruz. Tropical and Subtropical Agroecosystems 14: 807-818.

ICO (2017) International coffee agreement 2007 - Benefits of membership. International Coffee Organization. London. http://www.ico.org/Benefits_ICA2007.asp?section=About_Us. Fecha de consulta 5 de junio de 2017.

INEGI (1984) Cartas de efectos climáticos regionales (Primer y segundo periodo). Huixtla, Chiapas. Instituto Nacional de Estadística, Geografía e Informática. Aguascalientes, México. 1p

INEGI (2008) Conjunto de datos vectoriales. Unidades climáticas. Instituto Nacional de Estadística, Geografía e Informática. Aguascalientes, México. 1p

INEGI (2015) Cartas de uso del suelo y vegetación. Huixtla, Chiapas. Instituto Nacional de Estadística, Geografía e Informática. Aguascalientes, México. 1p

Jaffee D (2014) Brewing justice: Fair trade coffee, sustainability, and survival. University of California Press. California, USA. 331p.

Jolliffe I (2002) Principal component analysis. Springer. New York, USA. 487p.

Kass G (1980) An exploratory technique for investigating large quantities of categorical data. Applied Statistics 29: 119-127.

Kawulich B (2005) La observación participante como método de recolección de datos. Forum: Qualitative Social Research 2: 1-32.

Lewin B, Giovannucci D, Varangis P (2004) Coffee markets: New paradigms in global supply and demand. The World Bank. Washington DC, USA. 150p.

Lewis JM (2005) Strategies for survival: migration and fair trade-organic coffee production in Oaxaca, México. Center for comparative immigration studies. University of California, San Diego. California, USA. 82p.

López-López E, Caamal-Cauich I (2009) Los costos de producción del café orgánico del estado de Chiapas y el precio justo en el mercado internacional. Revista Mexicana de Economía Agrícola y de los Recursos Naturales 2: 175-198.

Lyon S, Bezaury J, Mutersbaugh T (2010) Gender equity in fairtrade-organic coffee producer organizations: Cases from Mesoamerica. Geoforum 41: 93-103.

Martínez-Torres M (2004) Survival strategies in neoliberal markets: peasant organizations and organic coffee in Chiapas. In: Otero G (ed). Mexico in transition: Neoliberal globalism, the state and civil society. California, USA. pp: 169-185.

Mas A, Dietsch T (2003) An index of management intensity for coffee agroecosystems to evaluate butterfly species richness. Ecological Applications 13: 1491-1501. 
Méndez V, Bacon C, Olson M, Petchers S, Herrador D, Carranza C, et al. (2010) Effects of fair trade and organic certifications on small-scale coffee farmer households in Central America and Mexico. Renewable Agriculture and Food Systems 25: 236-251.

Moguel P, Toledo VM (1999) Biodiversity conservation in traditional coffee systems of Mexico. Conservation Biology 13: 11-21.

Naylor L (2017) Auditing the subjects of fair trade: Coffee, development, and surveillance in highland Chiapas. Environment and Planning D: Society and Space 35: 816-835.

Nielsen $\varnothing$ J, Rayamajhi S, Uberhuaga P, Meilby H, Smith-Hall C (2013) Quantifying rural livelihood strategies in developing countries using an activity choice approach. Agricultural Economics 44: 57-71.

Nigh R (2010) Construcción de redes en la cadena de valor alimentaria. La agricultura campesina en Chiapas del siglo XXI. En: Fletes-Ocon H (Ed.) Pequeños productores y vulnerabilidad global agroalimentaria. Chiapas, México. pp: 38-60.

OECD (2008) Handbook on constructing composite indicators: Methodology and user guide. Organization for Economic Co-operation and Development. Paris, Francia. 162p

Parmentier S (2014) Scaling-up agroecological approaches: what, why and how? Oxfam-Solidarity. Bruselas, Bélgica. 92p

Pérez-Portilla E, Bonilla-Cruz S, Hernández-Solábac J, Partida-Sedas J (2011) Estrategia de mejoramiento de la producción cafetalera de la organización Campesinos Ecológicos de la Sierra Madre de Chiapas: caracterización de la bebida de café. Revista de Geografía Agrícola 47: 7-18.

Rosales-Martínez V, Martínez-Dávila J, Platas-Rosado D, Rosendo-Ponce A, Córdova-Ávalos V (2014) Cambio tecnológico en los agroecosistemas por migración familiar: el caso del municipio de Jamapa, Veracruz. Ecosistemas y Recursos Agropecuarios 1: 1-8.

Ruiz-Martinez I, Marraccini E, Debolini M, Bonari E (2015) Indicators of agricultural intensity and intensification: a review of the literature. Italian Journal of Agronomy 10: 74-84.

Rus D, Rus J (2014) Trapped behind the lines: The impact of undocumented migration, debt, and recession on a Tsotsil Community of Chiapas, Mexico, 2002-2012. Latin American Perspectives 41: 154-177.

Sánchez-Juárez G (2015) Los pequeños cafeticultores de Chiapas organización y resistencia frente al mercado. Universidad de Ciencias y Artes de Chiapas. Colección Thesis, Número 4. Chiapas, México. 225p

Sokal R, Michener C (1958) A statistical method for evaluating systematic relationships. University of Kansas Science Bulletin 38: 1409-1438.

Soto-Pinto L, Romero-Alvarado Y, Caballero-Nieto J, Segura G (2015) Woody plant diversity and structure of shade-grown-coffee plantations in Northern Chiapas, Mexico. Revista de Biología Tropical 49: 977-987.

Vellema W, Buritica A, Gonzalez C, D'Haese M (2015) The effect of specialty coffee certification on household livelihood strategies and specialisation. Food Policy 57:13-25. 
